\title{
Feasibility of closed-loop controller for righting seated posture after spinal cord injury
}

\author{
Julie O. Murphy, BSE; ${ }^{1-2 *}$ Musa L. Audu, PhD; ${ }^{1}$ Lisa M. Lombardo, MPT; ${ }^{2}$ Kevin M. Foglyano, BSE; ${ }^{2}$ Ronald \\ J. Triolo, $\mathbf{P h D}^{2-3}$ \\ ${ }^{1}$ Department of Biomedical Engineering, Case Western Reserve University, Cleveland, OH; ${ }^{2}$ Louis Stokes Cleveland \\ Department of Veterans Affairs Medical Center, Cleveland, $\mathrm{OH} ;{ }^{3}$ Department of Orthopaedics, Case Western Reserve \\ University, Cleveland, $\mathrm{OH}$
}

\begin{abstract}
Spinal cord injury (SCI) can compromise the ability to maintain an erect seated posture. This study examined the feasibility of a sensor-based threshold controller to automatically modulate stimulation to paralyzed hip and trunk extensor muscles to restore upright sitting from forward leaning postures. Forward trunk tilt was estimated from the anterior-posterior component of gravitational acceleration sensed by a sternum-mounted wireless accelerometer. Stimulation increased if trunk tilt exceeded a specified flexion threshold and ceased once upright sitting was resumed. The controller was verified experimentally in five volunteers with SCI and successfully returned all subjects to upright postures from forward leaning positions. Upper-limb effort exerted while returning to erect posture was significantly reduced (to $7.4 \%+/-$ $3.7 \%$ of body mass) pooled across all volunteers while using the controller compared with using continuous and no stimulation $(p<0.03)$. Controller response times were consistent among subjects when applied while sitting with $(0.30+/-0.05 \mathrm{~s})$ or without $(0.34+/-0.11 \mathrm{~s})$ a backrest. The controller enabled volunteers to lean farther forward $\left(59.7^{\circ}+/-16.4^{\circ}\right)$ in wheelchairs without upper-limb effort than with no stimulation. Clinical utility of the system for facilitating reach or preventing falls remains to be determined in future studies.
\end{abstract}

Key words: closed-loop controller, feedback control, functional electrical stimulation, functional neuromuscular stimulation, neuroprosthesis, SCI, seated posture, spinal cord injury, trunk stability, wireless accelerometer.

\section{INTRODUCTION}

After a midthoracic or higher level spinal cord injury (SCI), many individuals lose the ability to use their trunk and hip muscles to position and stabilize their torsos. The inability to control the motions of the spine and pelvis can severely compromise upper-limb workspace [1], increase the risk of pressure sore development [2], and adversely affect manual wheelchair propulsion and completion of many activities of daily living [3-4]. Because of this, individuals with SCI rank restoration of trunk stability as one of the highest priorities to enhance quality of life, regardless of injury level [5]. People with a high thoracic level SCI will often use their upper limbs or other nonpostural muscles to maintain an upright, seated posture [6]. Another strategy for compensating for paralysis of the core hip and trunk muscles is to adopt a "C"-shaped

\footnotetext{
Abbreviations: AIS $=$ American Spinal Injury Association Impairment Scale; ANOVA = analysis of variance; $\mathrm{C}=$ cervical; ECU = external command unit; FES = functional electrical stimulation; $\mathrm{L}=$ lumbar; $\mathrm{PID}=$ proportional, integral, derivative; $\mathrm{SCI}=$ spinal cord injury; $\mathrm{T}=$ thoracic.

*Address all correspondence to Julie O. Murphy, BSE; Louis Stokes Cleveland VA Medical Center, 10701 East Blvd, Mail Stop 151 W/APT, Cleveland, OH 44106-1702; 216-791-3800, ext 3230; fax: 216-231-3433.
}

Email: julie.murphy@case.edu

http://dx.doi.org/10.1682/JRRD.2013.09.0200 
kyphotic posture, which achieves static stability by shifting the center of mass of the trunk backward within the base of support [7-8]. However, this posture can contribute to chronic health problems such as reduced ventilation and increased pressure on bony prominences, internal organs, and intervertebral discs [9-10] that can lead to pressure sores, skeletal deformities, and back pain [1113]. To reduce the risk of falling forward out of their wheelchairs, individuals with SCI often wear chest straps or belts mounted to their backrests. However, such seating adaptations can prevent forward movement of the trunk and therefore compromise completion of many activities of daily living that require reaching forward. Another way to improve stability is to tilt the wheelchair seat backward, which may also impede the ability to reach forward [14].

Stiffening the otherwise paralyzed trunk and hip extensor muscles with continuous electrical stimulation can correct kyphotic seated posture, expand bimanual workspace, improve ventilation, alter interface pressures, statically stabilize the trunk, and improve manual wheelchair propulsion efficiency [1,15-18]. However, this strategy substitutes one statically stable posture for another. Upper-limb effort is still required to stabilize the body during transitions between unstimulated and stimulated postures and to maintain balance or restore erect sitting when presented with internally generated or externally applied perturbations. In a preliminary study, Vanoncini et al. found that closed-loop surface stimulation of only the trunk extensors using a proportional, integral, derivative (PID) controller or a linear quadratic regulator improved the stability of the trunk while in a single static posture in the presence of external disturbances [19].

The purpose of this study was to begin to address the issues related to adopting and maintaining a stable seated posture by exploring the feasibility of control systems that automatically modulate stimulation to recruit appropriate muscle synergies to restore erect sitting in the anterior-posterior direction. Such control systems should obviate the need for chest straps and upper-limb effort to maintain upright sitting, improve the ability to interact with the environment, further extend bimanual reach, and respond to potentially destabilizing disturbances that might otherwise result in a fall. This study specifically explored the design, technical performance, and clinical implementation of a sensor-based perturbation rejection controller to restore an upright, seated posture from a forward flexed position. The performance of this controller was compared with continuous open-loop stimulation and no stimulation conditions for a seated individual with SCI using functional electrical stimulation (FES) of the trunk and hip muscles. Each condition was tested in terms of its effect on upper-limb effort required to maintain upright, seated posture.

\section{METHODS}

\section{Participants}

Five volunteers with SCI resulting in paralysis of the hip and trunk extensor muscles participated in the study (Table 1). The volunteers ranged in age from 41 to $60 \mathrm{yr}$ (mean $52.4 \mathrm{yr}$ ), in weight from 54.4 to $82.5 \mathrm{~kg}$ (mean $69.7 \mathrm{~kg}$ ), and in height from 167.6 to $181.6 \mathrm{~cm}$ (mean $173.2 \mathrm{~cm})$. Two volunteers had sustained lower cervical (C6/C7), two mid-thoracic (T5/T6), and one lower thoracic (T10) injuries. Two of the volunteers had sensory/ motor complete (American Spinal Injury Association Impairment Scale [AIS] A), two sensory incomplete/ motor complete (AIS B), and one motor/sensory incomplete (AIS C) neurological deficits [20].

Table 1.

Summary of clinical characteristics of participants in this study.

\begin{tabular}{|c|c|c|c|c|c|c|c|c|c|}
\hline Subject & $\begin{array}{l}\text { Age } \\
\text { (yr) }\end{array}$ & Sex & $\begin{array}{l}\text { Height } \\
\text { (cm) }\end{array}$ & $\begin{array}{l}\text { Weight } \\
\text { (kg) }\end{array}$ & $\begin{array}{l}\text { Injury } \\
\text { Level }\end{array}$ & $\begin{array}{c}\text { AIS } \\
\text { Grade* }\end{array}$ & $\begin{array}{c}\text { Time Post } \\
\text { Injury }^{\dagger}(\mathrm{yr})\end{array}$ & $\begin{array}{c}\text { Time Post } \\
\text { Implant }^{\dagger}(y r)\end{array}$ & Muscles Stimulated \\
\hline$\overline{\mathrm{S} 1}$ & 41 & $\mathrm{~F}$ & 167.6 & 54.4 & $\mathrm{C} 7$ & $\mathrm{C}$ & 14.6 & 12.9 & ES, QL, GM, SM, AM \\
\hline $\mathrm{S} 2$ & 56 & M & 175.3 & 82.5 & $\mathrm{C} 7$ & B & 5.3 & 1.5 & ES, QL, IL, GM, SM, AM \\
\hline S3 & 57 & $\mathrm{~F}$ & 167.6 & 68.6 & T5-6 & B & 9.4 & 5.0 & ES, GM, SM, AM, GMed \\
\hline S4 & 60 & M & 174.0 & 77.7 & $\mathrm{~T} 6$ & A & 14.5 & 7.2 & ES, GM, SM, AM \\
\hline S5 & 48 & M & 181.6 & 65.5 & $\mathrm{~T} 10$ & A & 6.6 & 1.7 & ES, GM, SM, AM \\
\hline
\end{tabular}

${ }^{*} \mathrm{~A}=$ motor and sensory complete, $\mathrm{B}=$ motor complete with sensory sparing, $\mathrm{C}=$ motor and sensory incomplete.

${ }^{\dagger}$ At time of initial study enrollment and testing.

$\mathrm{AM}=$ posterior portion of adductor magnus, $\mathrm{C}=$ cervical, $\mathrm{ES}=$ erector spinae, $\mathrm{F}=$ female, $\mathrm{GM}=$ gluteus maximus, $\mathrm{GMed}=\mathrm{gluteus}$ medius, $\mathrm{M}=$ male, $\mathrm{QL}=\mathrm{qua}-$ dratus lumborum, $\mathrm{S}=$ subject, $\mathrm{SM}=$ semimembranosus, $\mathrm{T}=$ thoracic. 
All volunteers received implanted neuroprostheses for standing, transfers, or exercise during their participation in other studies. Participants had an 8-channel implanted receiver-stimulator [21] system for standing transfer [22-24] and/or a 16-channel implanted stimulator-telemeter [25] system to study standing balance [2628] or peripheral nerve cuff electrodes [29-30]. Both the 8- and 16-channel stimulators delivered constant current, charge-balanced biphasic stimulus waveforms. Stimulation parameters were adjusted as necessary with amplitude ranging from 2 to $20 \mathrm{~mA}$ and pulse width ranging from 0 to $250 \mu \mathrm{s}$.

These implanted systems contained stimulators that were connected to 8 and/or 16 implanted electrodes. In all subjects, the lumbar (L) erector spinae were recruited with bilateral intramuscular stimulating electrodes [31] inserted near the L1-L2 spinal nerves [23,32-33] for trunk extension, and the gluteus maximus muscles were activated bilaterally with intramuscular electrodes or epimysial electrodes. For additional hip extension, the posterior region of the adductor magnus and the semimembranosus were stimulated bilaterally with intramuscular electrodes in four subjects. For additional medial-lateral stability, the quadratus lumborum was recruited bilaterally with intramuscular electrodes near the T12-L1 spinal nerves for two subjects, and the gluteus medius was activated bilaterally with intramuscular electrodes in one subject. The muscles stimulated for each subject are summarized in Table 1.

\section{Stimulation Patterns}

A continuous stimulation pattern was developed individually for each volunteer to be used in an open-loop manner. Pulse widths were tuned for each stimulation channel to maintain sagittal and coronal alignment of the trunk in a static, erect seated posture. This continuous pattern was generally used by the participants in their home environments [15,17-18]. For all subjects, stimulus pulse widths and amplitudes were chosen that would avoid interference with respiration or the discomfort sometimes associated with activating the abdominal muscles or sensory fibers near the stimulating electrode. This continuous stimulation was delivered at a frequency of $20 \mathrm{~Hz}$.

A different righting pattern was developed for the purposes of the current study. Stimulation pulse durations were adjusted for each user to allow the participant to return from a static, fully forward-flexed position to an upright, erect seated posture. To achieve this function, the stimulation frequency was increased to 25 or $30 \mathrm{~Hz}$ across all channels and pulse durations to the hip and back muscles were increased, over the continuous pattern, to maximal levels beyond which no further force recruitment was possible. Stimulation levels were reduced if the participant experienced any adverse sensation or undesired muscle spasms. Additional hip extensors not active in the continuous pattern were often activated for the righting maneuver.

\section{Controller Design}

A basic threshold-based closed-loop feedback control design was used to modulate stimulation automatically based on trunk tilt. A three-axis accelerometer embedded in an EZ430-Chronos sports watch (Texas Instruments; Dallas, Texas) was configured as a tilt sensor. Hardware, based on the CC430 microcontroller (Texas Instruments), was integrated into a wearable external command unit (ECU) [34], which powered and controlled the actions of the implanted stimulators and allowed the accelerometer output to be used as a feedback signal. The control algorithm was implemented in Simulink and XPC Target (MathWorks Inc; Natick, Massachusetts) software for real time control of trunk tilt. The accelerometer signal was sampled at $40 \mathrm{~Hz}$ and wirelessly streamed to the ECU. The watch was strapped around the chest of the subject and centered just above the xiphoid process as shown in Figure 1. The sensor in the watch was oriented such that the component of the acceleration due to gravity serving as the feedback control signal was located in the sagittal plane of the body and pointing in the direction normal to the sternum. This component of the sensor output can be correlated trigonometrically to the angle of the trunk, as diagramed in Figure 1. As the forward tilt of the user increases, the component of the acceleration due to gravity used as the feedback control signal also increases. Approximately $0^{\circ}$ of trunk tilt denoted an upright, erect posture. Positive tilt angles denoted a forward movement in the sagittal plane, while negative tilt angles denoted a backward movement.

The control scheme used to modulate stimulation was a simple two-threshold system, illustrated in Figure 2. Forward trunk flexion past a specified angle, designated as the "flexion threshold," triggered the stronger righting pattern of stimulation. This pattern would arrest the continued forward flexion of the trunk, extend the trunk, and return the user to an upright, erect seated posture. Once the trunk crossed another specified angle closer to the 


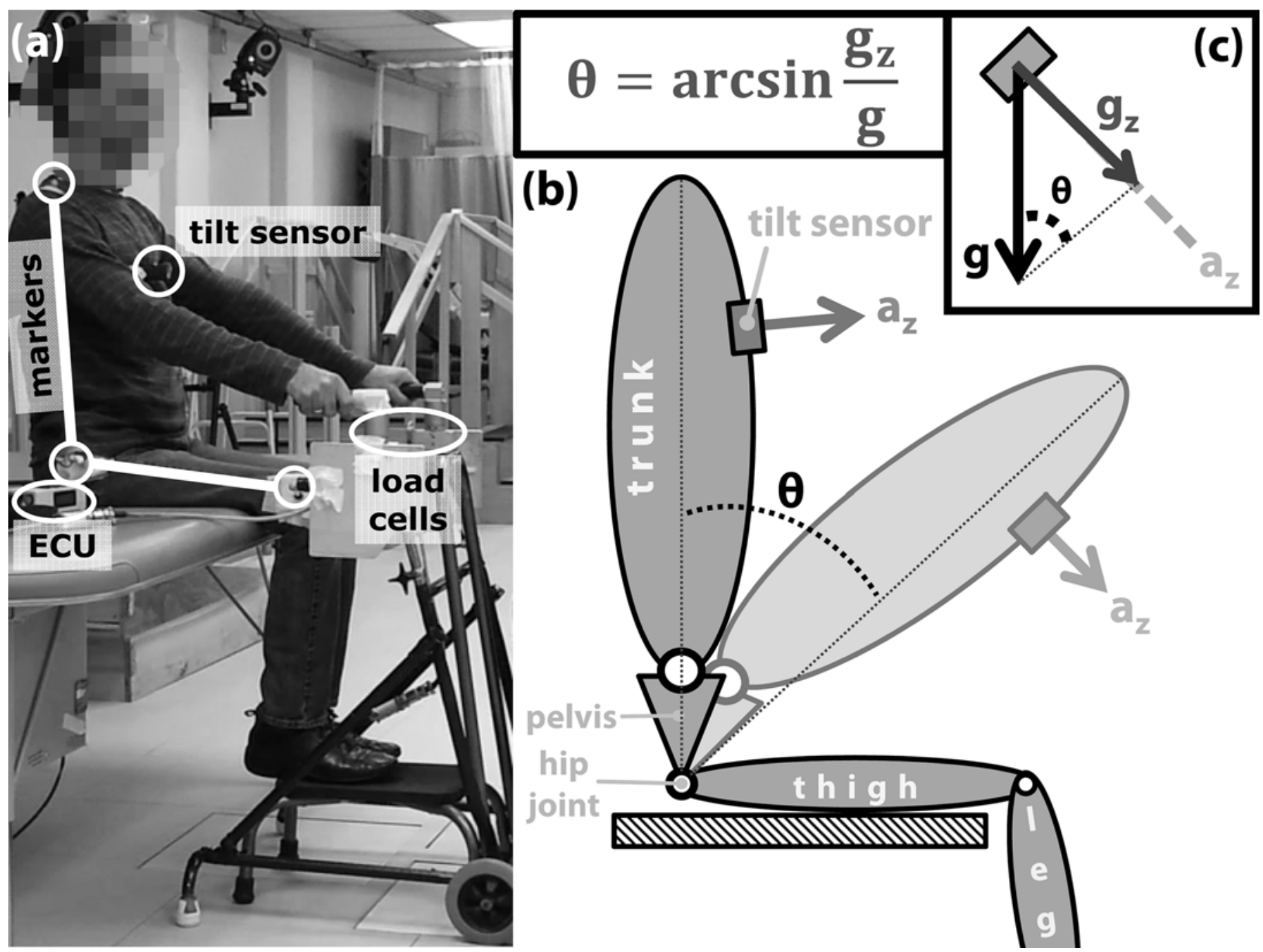

Figure 1.

Experimental setup for upper-limb loading trials. (a) Placement of tilt sensor, markers, and load cells; (b) definition of trunk angle ( $\theta$ ); and (c) geometric relationship between trunk angle and acceleration due to gravity measured with sensor. Under quasistatic conditions, $g_{z}$ constitutes majority of acceleration used as feedback signal. $a_{z}=$ component of accelerometer signal used as feedback signal, $\mathrm{ECU}=$ external command unit, $\mathrm{g}=$ acceleration due to gravity, $\mathrm{g}_{\mathrm{z}}=$ component of acceleration due to gravity measured by sensor.

erect position, termed the "upright threshold," the stimulation turned off completely. The upright threshold was set to approximately $7^{\circ}$ when the subject was seated upright with no stimulation. The flexion threshold was initially determined to be the point at which the users felt as though they would lose their ability to right themselves without additional assistance. It was adjusted for testing and comfort in consultation with the users after practice with the system. Table 2 contains the values of the thresholds used for each subject during the experi- ments. Both thresholds could be adjusted in real time, although the continuous and righting stimulation patterns were preprogrammed and consistent throughout the experiments.

\section{Upper-Limb Loading}

To quantify the performance of the control system, volunteers were seated on an adjustable mat table without a backrest, as shown in Figure 1. A walker with 6-degree of freedom load cells (MCW-1000, Advanced Mechanical 


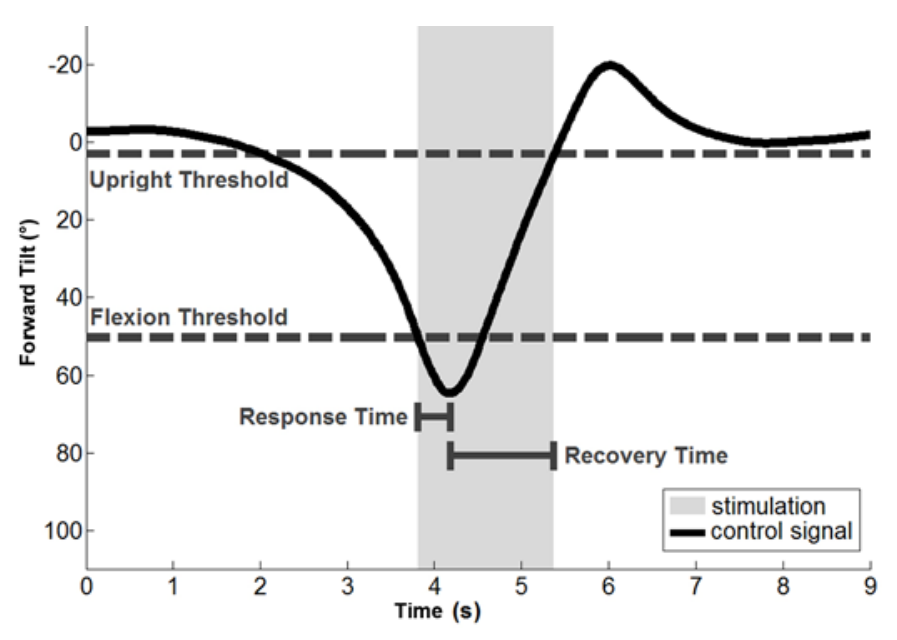

Figure 2.

Example of control signal from tilt sensor and thresholds used by controller to trigger stimulation. Stimulation is applied when control signal is below flexion threshold. Stimulation is ceased when control signal is above upright threshold. Note: Values on $y$-axes for all figures containing forward tilt are inverted to align with intuitive nature of movement.

Table 2.

Summary of threshold angles when controller was active during upper-limb loading trials on mat table and in wheelchair.

\begin{tabular}{lccccc}
\hline \multirow{2}{*}{ Subject } & \multicolumn{2}{c}{ Upper-Limb Loading $\left(^{\circ}\right)$} & & \multicolumn{2}{l}{ Wheelchair Trials $\left(^{\circ}\right)$} \\
\cline { 2 - 3 } \cline { 5 - 6 } & Upright & Flexion & & Upright & Flexion \\
\hline S1 & 5.3 & 42.9 & & 11.2 & 22.1 to 49.3 \\
S2 & 5.6 & 38.3 & & N/A & N/A \\
S3 & 14.6 & 51.4 & & -1.7 to 14.6 & 14.6 to 41.2 \\
S4 & 5.3 & 35.5 & & N/A & N/A \\
S5 & 3.0 & 37.8 & & -8.7 to 6.5 & 6.5 to 37.8 \\
\hline
\end{tabular}

Note: Flexion threshold values indicate when stimulation started and were not necessarily indicative of how far user actually flexed forward. Testing in wheelchair occurred at multiple threshold values to determine effectiveness of controller at various levels. Upright threshold was always set to lower angle than flexion threshold in order for controller to function properly.

$\mathrm{N} / \mathrm{A}=$ data not available for subject.

Technology Inc; Watertown, Massachusetts) integrated into its handles was placed in front of the subject. The load cell outputs were recorded at a sampling frequency of at least $120 \mathrm{~Hz}$. The level of the mat was adjusted such that the handles were at or below the femur of the subjects to allow them to comfortably lean forward during the experiment without falling. A physical therapist spotted the subjects during the experiment to ensure safety. The forces exerted by the user's upper limbs were recorded as a measure of the effort needed to return to an upright position. The experiments took place within the measurement volume of a 16-camera motion capture system (Vicon Motion Systems and Peak Performance Inc; Oxford, United Kingdom). Reflective markers were placed bilaterally on the acromion of the scapula, greater trochanter of the femur, and lateral epicondyle of the femur. Marker positions were sampled at $120 \mathrm{~Hz}$. Trunk angle was calculated as the angle between global vertical axis of the measurement volume and the line segment from the midacromion to mid-greater trochanter.

During each trial, the volunteers were instructed to allow their trunks to flex forward while resting their hands on the handles of the instrumented walker. They were asked to return to an upright posture using their hands as little as possible. The force recorded by the load cells, or upper-limb loading, was measured under three conditions: (1) with the controller active, (2) with only continuous stimulation, and (3) with no stimulation. For the conditions under which the subjects restored their posture volitionally, they were instructed to right themselves whenever their forward tilt crossed a specified angle that was similar to the flexion threshold of the controller. Because all subjects were able to right themselves volitionally using their upper limbs, all three conditions resulted in a maneuver similar to the one shown in Figure 2. Before conducting any trials used for analysis, each subject was allowed to practice this maneuver under each condition to ensure repeatable operation without any confounding training effects. Most subjects only needed to practice two to three times to feel comfortable with the action of the controller. Then, the maneuver was repeated for 10 to 20 trials for each condition. To minimize effects of muscle fatigue, $10 \mathrm{~s}$ breaks were given between trials, and longer 5 min breaks were given between conditions. Individual trials for each stimulation condition were tested in succession, but the order in which the stimulation conditions were tested was randomized for each subject.

The technical performance of the controller was quantified in terms of response and recovery times (Figure 2). The response time of the controller was defined as the difference between the time when the trunk first crossed the flexion threshold (onset of stimulation) and the time when the forward movement was arrested and the trunk motion began to reverse toward the erect posture. The recovery time of the controller was defined as the difference between the time when the peak trunk flexion angle occurred and the 
time when the trunk angle crossed the upright threshold to terminate stimulation, as shown in Figure 2. The response and recovery times were reported only during the trials for which the controller was active to quantify controller performance. The mean and standard deviation of these times were calculated for each subject. The times for each trial were also pooled across all subjects, and the overall mean and standard deviation were computed.

Functional implications of the stimulation conditions were quantified in terms of the upper-limb effort exerted on the instrumented walker during the recovery phase of the maneuver. In order to compare the amount of effort that the participants exerted while righting themselves under the three conditions, the mean of the upper-limb loading was calculated during the recovery time described previously. The three-dimensional force vector magnitude was calculated for three subjects, but because the vertical component of the magnitude constituted approximately 93 percent of the resultant, only the vertical component was used in upper-limb effort calculations for all five subjects. The vertical components from both load cells in the handles of the walker were summed together. The force was zeroed before each trial with subjects sitting erect to account for the passive weights of the upper limbs resting on the load cells. The absolute values of the vertical forces were taken into account for the potential of negative due to a subject pulling up on the load cells. Then, the mean of the net force exerted by the user during the recovery time was calculated for each trial. For each subject, the mean upper-limb forces were normalized by body mass, averaged together, and a standard deviation among the trials was calculated for each condition. The normalized means from each trial were pooled together for each specific condition, and the average normalized upper-limb effort and standard deviation across all subjects were computed.

The effect of the stimulation condition on the average normalized upper-limb effort was tested for significance using a generalized linear analysis of variance (ANOVA). The three stimulation conditions were incorporated as fixed factors, and the subjects were set as random factors in the model. The normalized upper-limb effort averaged across each condition for each subject was set as the response of the system. The residuals obtained from the ANOVA model were checked with the Anderson-Darling test for normality and the Bartlett test for equal variances. For each participant, multiple independent two-sample $t$ tests with unequal variances and sample sizes were used to test for significant differences between the average normalized upper-limb efforts among the different conditions.

\section{Testing in Wheelchair}

To better approximate real-world conditions, the operation of the controller was evaluated with all subjects seated in their own wheelchairs. Volunteers were located in the same motion capture measurement volume described previously with the same marker placement. Before each trial, they were asked to sit up straight using their arms. With the controller active, they were instructed to lean forward with their arms crossed over their chests or resting on their backs. Subjects leaned forward until they crossed the flexion threshold, and the stimulation returned them to an upright posture against the backrests of their wheelchairs. This maneuver was repeated approximately 30 times. Participants were given $10 \mathrm{~s}$ rest breaks between trials, and 5 min rest breaks were given after every 10 trials or as needed. Operation of the controller was tested by adjusting the flexion threshold of the controller to different levels. Trials were not used in data calculations if the subject employed additional compensatory mechanisms such as rapidly retracting the arms, extending the neck, or pushing up against the thighs during the righting maneuvers. All five subjects tested the controller in their own wheelchairs, and full data sets suitable for analysis were recorded for three of the five subjects (S1, S3, S5).

Response and recovery times were calculated while the controller was active using the same method described in the upper-limb loading trials. The peak forward flexion angle achieved by the subjects with the controller active was set as the angle at which the controller would arrest their forward motion before their upper body touched their thighs and returned them back to an erect sitting posture without additional compensatory mechanisms. This angle was compared with the peak forward flexion angles that the subjects determined to which they could lean forward and still return to an erect posture without assistance under continuous and no stimulation conditions. All forward flexion angle measurements were calculated using the trunk angle measured by the markers using motion capture system.

To analyze the performance of the accelerometer as a tilt sensor, forward tilt was estimated by converting the control signal of the accelerometer to degrees using the trigonometric relationship between the trunk angle and the acceleration due to gravity as shown in Figure 1. 
Then the signal was calibrated to account for the sensitivity and the offset of the sensor at the beginning of each experiment [35-36]. This calibration enabled the control signal to be compared with the trunk angle calculated from the motion capture markers.

\section{RESULTS}

The controller returned all users to an upright posture quickly enough to prevent a fall without causing discomfort. For conditions requiring voluntary effort, all participants were able to lower and push themselves up in trajectories that matched the actions of the controller. Testing sessions contained both upper-limb loading and wheelchair trials and lasted up to $4 \mathrm{~h}$. No adverse events occurred during testing. On occasion while testing in the wheelchair, the righting pattern succeeded in arresting the forward motion of the trunk, but it could not return the user to an upright posture without additional compensatory mechanisms. These trials were not used in further analysis. After 3 to $5 \mathrm{~min}$ of rest, the original function of the righting pattern was restored and testing resumed.

\section{Upper-Limb Loading}

The flexion and upright threshold values used for each participant during the upper-limb loading tests on the mat are listed in Table 2 . Across all participants, the average flexion threshold was $41.2^{\circ} \pm 6.3^{\circ}$, and the average upright threshold was $6.8^{\circ} \pm 4.5^{\circ}$. Figure 3 shows the timing results while the controller was active during the upper-limb loading trials for each subject as well as across all subjects. The average response time across all the subjects was $0.30 \pm 0.05 \mathrm{~s}$, ranging from 0.23 to $0.35 \mathrm{~s}$ while the standard deviations of the individual response times ranged from 0.03 to $0.04 \mathrm{~s}$. The average recovery time across all of the subjects was $1.10 \pm 0.41 \mathrm{~s}$, ranging from 0.48 to $1.30 \mathrm{~s}$. The standard deviations of the individual recovery times ranged from $0.05 \mathrm{~s}$ to $0.55 \mathrm{~s}$.

Figure 4 shows the normalized upper-limb loading results for each subject and condition as well as across all subjects. Overall, upper-limb loads equivalent to $16 \pm$ 6.0 percent of body weight were exerted to recover from a forward flexed position with no stimulation over 64 trials. With continuous stimulation, however, upper-limb loading decreased to $12.8 \pm 6.5$ percent body weight over 65 trials. When the controller was active, upper-limb effort was further reduced to $7.4 \pm 3.7$ percent of body weight over 67 trials. The ANOVA results from the pooled data indicated that the stimulation condition with the controller active led to average normalized upper-limb forces that were significantly different $(p<0.03)$ from the average normalized upper limb forces obtained from the continuous and no stimulation conditions. The residuals were consistent with the normal distribution, and the variances of the residuals from the three conditions were not statistically different. Using two-sample $t$-tests for each subject, the average normalized upper-limb effort between each stimulation condition was significantly different from the subject's upper-limb effort under the other conditions $(p<$ 0.02 ), except for two cases. There was no significant difference between no stimulation and continuous stimulation for subject S4 $(p>0.48)$, although upper-limb loading with the controller active was significantly different from both of those conditions. Similarly, no significant difference was observed between continuous stimulation and controller active conditions for subject S5 $(p>0.48)$, although the two stimulation conditions both resulted in significantly less upper-limb loading than with no stimulation. In all cases, at least one of the forms of hip and trunk stimulation (continuous or active controller) was always superior to the no stimulation condition.

\section{Testing in Wheelchair}

The controller was able to return five out of five subjects to an upright, erect seated position in their own wheelchairs without any additional compensatory mechanisms. A typical trial from one of the subjects ( 11$)$ is

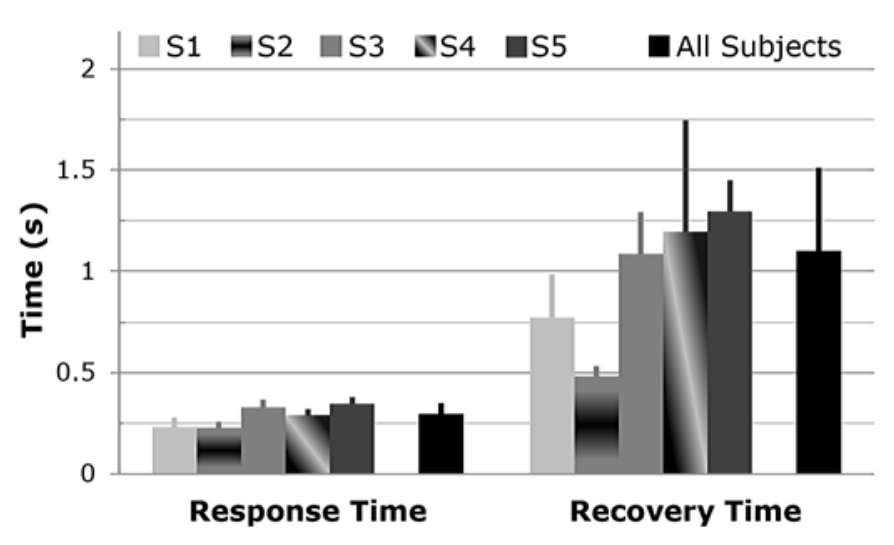

Figure 3.

Average response and recovery times for each subject, with standard deviation, when threshold-based controller was active during upper-limb loading trials with hands placed on handles of instrumented walker. $\mathrm{S}=$ subject. 
shown in Figure 5. In the top trace, she attempted to lean forward approximately halfway between her upright posture and a fully flexed posture. Without stimulation of her hip and trunk muscles, her upper body fell forward onto the top of her legs. The compensatory mechanisms she employed to sit back including extending her head and neck and retracting her arms and shoulders are evident as short, intermittent extension angles in the tilt signal. When the controller was active (Figure 5(b)) with a threshold set to approximately $42^{\circ}$, she was able to lean forward approximately $69^{\circ}$ before the higher level of stimulation automatically returned her to an erect seated posture.

At the peak flexion angle shown in Figure 5 for subject S1, the difference between the angle measured by the accelerometer and the angle measured by the motion capture system during the wheelchair trials was approximately $23^{\circ}$. Across all 78 wheelchair trials of the three subjects with complete data sets, the average difference between the actual trunk angle and tilt sensor approximation was $7.6^{\circ} \pm 8.7^{\circ}$ and the median difference was $4.6^{\circ}$.

The ranges of flexion and upright threshold values used during the wheelchair testing are listed in Table 2. For flexion thresholds that were closer to upright, the upright threshold was adjusted to allow the user to lean backward slightly in order to ensure a separation between the two thresholds and avoid limit cycling. Figure 6 shows the timing results with the controller active during

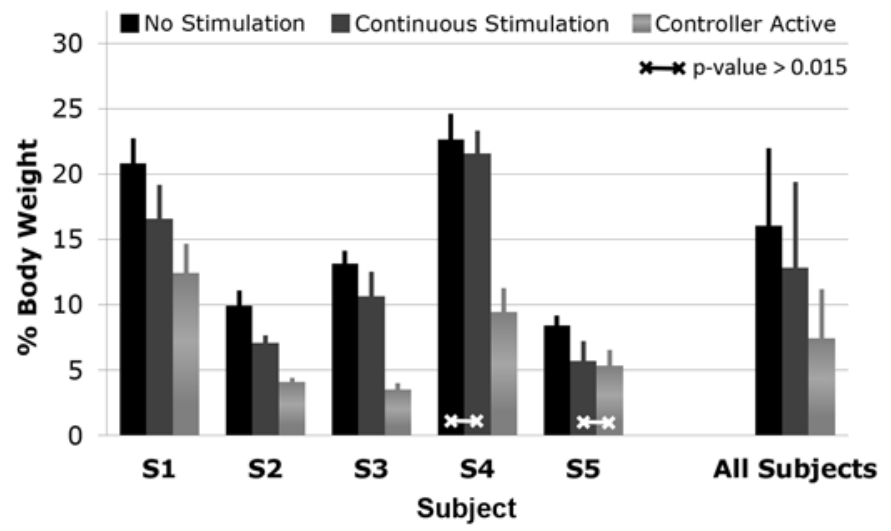

Figure 4.

Average upper-limb loading, with standard deviation, normalized to each subject's body mass, during recovery time for each condition (righting pattern with controller active, continuous stimulation, and no stimulation). Only two comparisons among each subject were not statistically significant, as denoted in graph, with $p>0.015$.
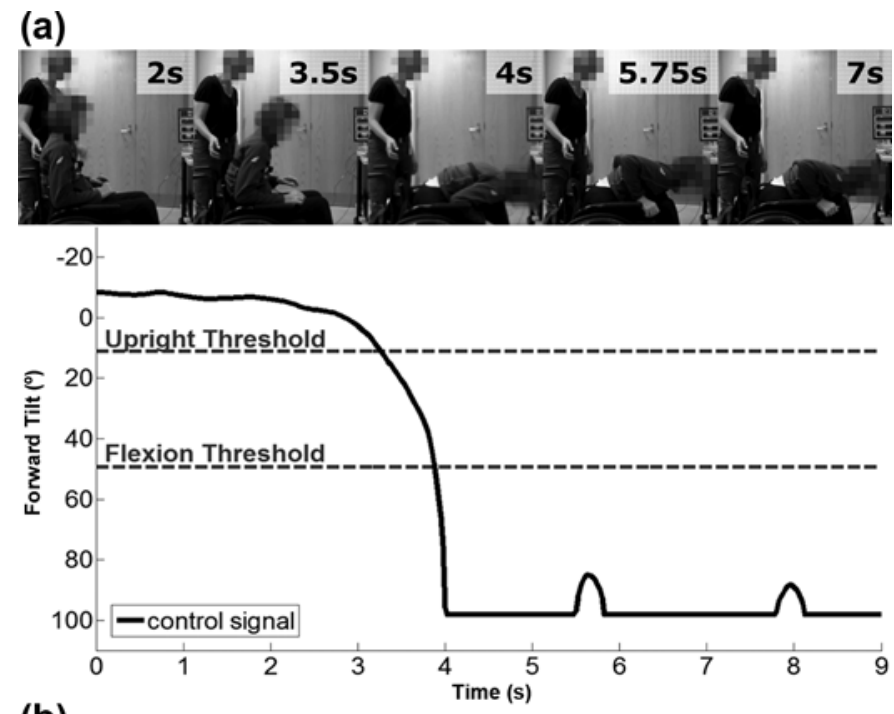

(b)

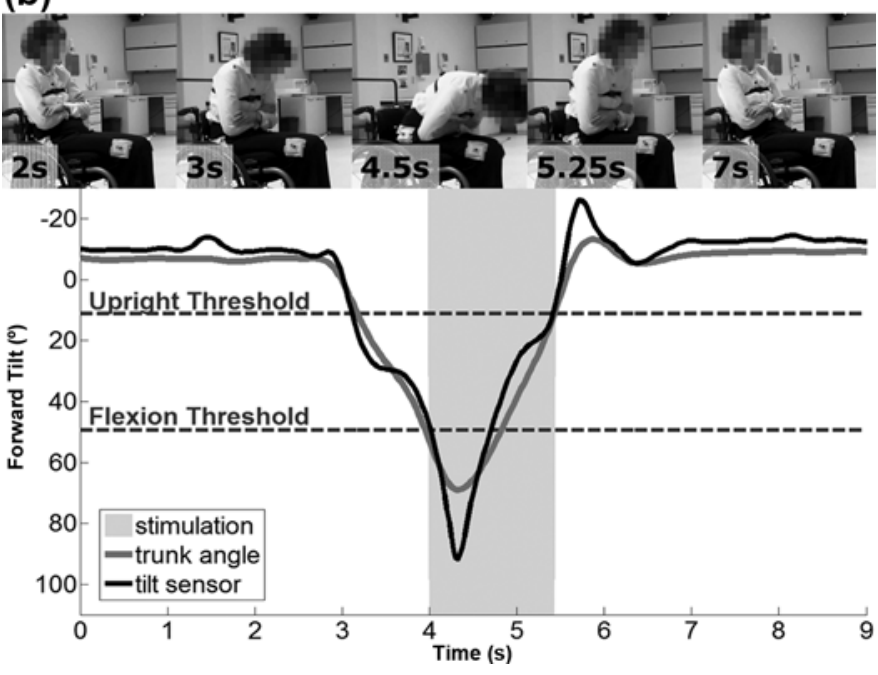

Figure 5.

Forward trunk flexion and attempts of subject S1 to resume upright sitting in wheelchair (a) with no stimulation and (b) with threshold-based controller active. Actual and accelerometerbased estimate of trunk angle are shown.

the wheelchair trials. The average response time over 78 trials across the three subjects was $0.34 \pm 0.11 \mathrm{~s}$, ranging from 0.32 to $0.36 \mathrm{~s}$, while the standard deviation of the individual response times ranged from 0.07 to $0.16 \mathrm{~s}$. The average recovery time across all of subjects was $1.20 \pm$ $0.37 \mathrm{~s}$, ranging from 1.09 to $1.31 \mathrm{~s}$, while the standard deviation of the individual recovery times ranged from 0.33 to $0.41 \mathrm{~s}$.

The peak forward trunk flexion angle achieved in the wheelchair with the controller active averaged approximately $59.7^{\circ} \pm 16.4^{\circ}$ (Table 3 ). With the controller 


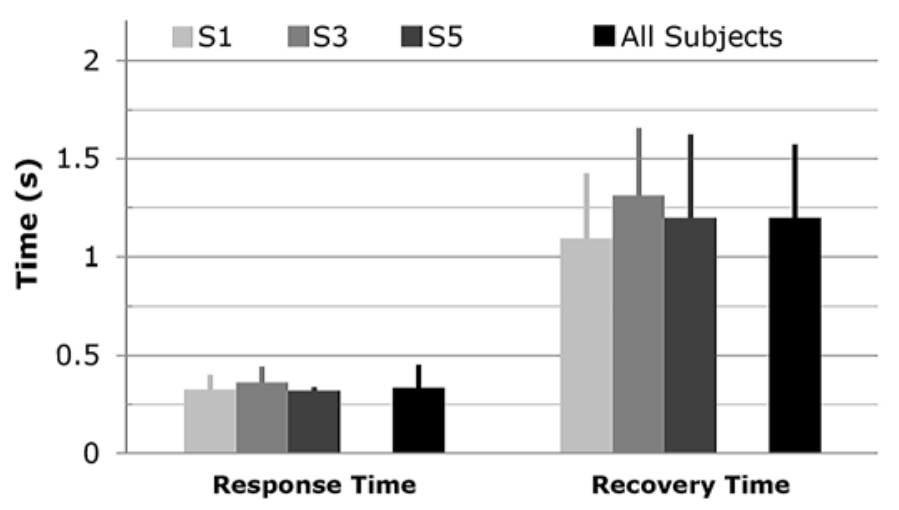

Figure 6.

Average response and recovery time for each subject, with standard deviation, when threshold-based controller was active during wheelchair trials while arms were crossed on their chest or resting on their back.

Table 3.

Summary of peak forward flexion angles of trunk under three conditions.

\begin{tabular}{lccc}
\hline Subject & $\begin{array}{c}\text { No } \\
\text { Stimulation } \\
\left({ }^{\circ}\right)\end{array}$ & $\begin{array}{c}\text { Continuous } \\
\text { Stimulation } \\
\left.\mathbf{(}^{\circ}\right)\end{array}$ & $\begin{array}{c}\text { Active } \\
\text { Controller } \\
\left({ }^{\circ}\right)\end{array}$ \\
\hline S1 & N/A & N/A & 73.1 \\
S3 & 20.4 & 21.4 & 64.7 \\
S5 & 38.6 & 66.3 & 41.4 \\
\hline Note: Larger angle indicates that user was able to achieve more forward flexion \\
than smaller angles. \\
N/A = data not available for subject. \\
\hline \hline
\end{tabular}

active, one subject (S3) reached an angle of almost $40^{\circ}$ lower than with continuous or no stimulation. Another subject (S5) achieved his lowest angle with continuous stimulation. As with the upper-limb effort on the mat table, one of the two stimulation conditions always allowed the users to assume a more flexed trunk position and return to upright than volitional function alone with no stimulation. Data for the peak forward trunk flexion angle under the continuous and no stimulation conditions were not recorded for subject $\mathrm{S} 1$.

\section{DISCUSSION}

This study demonstrated the feasibility of implementing a simple threshold-based control scheme to restore movement of the trunk for individuals with lower cervical or thoracic level SCI. The controller worked consistently across all five subjects in this study on a mat table and in their own wheelchairs under laboratory conditions. It is important to note that the controller was not designed to facilitate movement to a desired set point nor maintain a specific seated posture away from nominal sitting. It was designed to detect a forward fall and return the user to an erect, upright, seated posture. A more complex controller and additional muscles may be necessary to stabilize the trunk during movements in other orientations and postures due to increased center of mass displacement when perturbations are applied diagonally compared with along the anterior-posterior or mediallateral directions [37]. Fujita et al. used a proportionalderivative controller to stabilize the trunk during stance and determined that stimulation of the medial-lateral trunk flexors might be required to stabilize the trunk in the coronal plane [38]. A qualitative feasibility study [19] already showed that, with the pelvis held fixed, modulating pulse duration during surface stimulation of the erector spinae using a PID controller could return the trunk to an erect posture after an external disturbance in the forward direction. Our approach allowed for rotation about both the hips and the lumbar back, which resulted in larger overall forward trunk tilt approaching $60^{\circ}$ (Table 3) compared with a peak value of approximately $20^{\circ}$ reported in Vanoncini et al. [19]. Stimulating hip extensors in addition to the trunk extensors with the thresholdbased system expanded the controllable trunk angle with short bursts of stimulation that may also have the potential to reduce or avoid muscle fatigue.

\section{Upper-Limb Loading}

Testing on the mat table without a backrest demonstrated that the controller could successfully trigger stimulation and cause a functional change in the user's trunk angle quickly enough to prevent a forward fall. As seen in the standard deviations, this response time was relatively consistent for all trials within each subject as well as across all subjects. This implies that the controller action was repeatable, predictable, and reliable. The controller comfortably returned the users to upright postures quickly enough to prevent a fall while still avoiding excessive jerking or causing them to fall backward onto the mat table. Recovery times were more variable because they depend on factors such as distance between the two thresholds and the strength of the stimulated contractions elicited by the righting pattern. This pattern was customized for each volunteer and was highly subjectspecific since each individual electrode had unique 
contractile and recruitment properties. In addition, the implanted systems were optimized for bipedal functions specific to other studies (such as standing). Thus, erect seated posture was often a secondary benefit and not the primary goal of the original implanted systems. Future studies with a muscle set optimized for trunk function would allow a more thorough exploration of the potential benefits and limitation of the threshold-based controller or other systems to set and maintain seated posture and balance. Based on our results, this control scheme should be applicable to any FES system that results in sufficient muscle force to return the trunk to an erect posture, including percutaneous or surface stimulation.

Across all subjects, analysis of the upper-limb effort showed that any type of stimulation significantly reduces the amount of force exerted by the arms to return to an upright posture. Stronger stimulated responses or different stimulation patterns may reduce the upper-limb effort even further. Although this study focused on characterizing the ability of the controller to right the user during a forward fall, the reduction in upper-limb effort suggests that a threshold-based control scheme may also enable performance of bimanual tasks that were difficult or impossible without stimulation. With trunk and hip stimulation arresting forward lean and returning the user to upright, the upper limbs would be free to pick up objects from the floor or perform other bimanual activities. Evaluating these potential functional outcomes is beyond the scope of this study, and further testing would be important to determine the clinical relevance of using this threshold-based control scheme.

As shown in Figure 4, continuous stimulation did not significantly reduce upper-limb effort for S4, but remained consistent with the effort exerted during the trials with no stimulation. However, a significant reduction in the upper-limb effort required to restore an erect posture was seen with the controller active. This implies that his continuous stimulation pattern may have improved his posture by stiffening his trunk rather than extending it. For subject S5, there was no significant difference between continuous stimulation and the threshold-based controller, but both types of stimulation significantly reduced the amount of upper-limb effort when compared with no stimulation. This may be because his muscles reached full recruitment at lower levels of stimulation and the righting pattern was unable to provide a sufficient increment in muscle force to produce a significant difference. In addition to variations in stimulated strength, the differences in upper-limb effort among subjects may be due to individual variations in injury level, residual voluntary trunk and hip function, the custom threshold values set for each volunteer, or personal habits and preferences for interacting with the instrumented walker.

\section{Testing in Wheelchair}

The threshold-based controller enabled all of the subjects to right themselves in their own wheelchairs without any upper-limb effort, intentional interventions to activate stimulation, or compensatory mechanisms involving voluntary head or arm movements. The comparison between no stimulation and the stimulation activated by the controller for subject S1 in Figure 5 graphically illustrates the ability of the system to restore upright sitting automatically without relying on her hands or arms to manipulate switches or button; pushing up against her thighs or wheelchair armrests; or retracting her head, arms, and shoulders. Although the functional implications of such a control system during activities of daily living remain to be determined in future studies, these results suggest that the task-dependent modulation of stimulation to the hip and trunk muscles may enable forward reaching and completion of bimanual tasks. This would improve a user's ability to interact with and exert control over objects in the environment that may facilitate completion of many work or leisure-related tasks and activities of daily living. As with any new assistive device, the participants in this study required different amounts of time to feel comfortable with the operation of the new control system. Future work is needed to determine the best rehabilitation strategy for implementing this device for functional use.

Both the response and recovery times while sitting in the wheelchair (Figure 6) were within $100 \mathrm{~ms}$ of respective times while sitting on the mat without a backrest. Since the controller is most likely to be used while sitting in the wheelchair, these results indicated that the control scheme could work effectively to restore seated posture under real world conditions, even when users do not have the option of resting their hands on an assistive device such as the walker handles.

This control scheme may be more beneficial for individuals with higher-level injuries who have less intact voluntary control. For example, the peak forward trunk flexion angle of $64.7^{\circ}$ with the controller active achieved by subject S3, who had a T5-6 level injury, suggests that the controller may significantly extend sagittal reach and 
expand seated workspace (Table 3). Subject S5, however, had a T10 level injury and was able to accomplish more volitionally with no stimulation than S3 due to having more residual control over his trunk and hip muscles. This may also have contributed to the lack of difference between continuous stimulation and the threshold-based control of stimulation for other performance measures for S5. Individuals with incomplete injuries who have more volitional control over their trunk and hip muscles may generate similar results as S5. A larger sample size would be needed to fully investigate the interactions of injury level and degree of completeness with controller performance and to understand the user population most likely to benefit from trunk stimulation. The most forward flexed angles achieved volitionally with or without stimulation may also depend on the user's individual perception of safety as well as their innate fear of falling, which might have been minimized by practice with the hip and trunk stimulation system.

Using an accelerometer as a tilt sensor was an effective means of determining forward trunk tilt for use as a feedback signal. Under conditions in which the trunk was moving with increasing or decreasing speed, the accelerometer measured angles that were different from the actual position of trunk as measured by the motion capture system. The difference between the actual and accelerometer-based trunk angles can be seen at the peak flexion angle in Figure 5 when the trunk decelerated rapidly and reversed direction. The accelerometer-based trunk angle estimate exceeded the actual angle because of the added acceleration of the trunk to the component due to gravity. To compensate for this error, some applications use two accelerometers per body segment [39], simple low-pass filtering to remove high-frequency linear accelerations [40], or a combination of accelerometer and gyroscope signals [40-41]. In this study, however, the difference between the accelerometer estimate and actual trunk angle could be viewed as a safety factor because the stimulation would trigger sooner as the trunk accelerated with increasing trunk angle as it approached the flexion threshold. As indicated by the large standard deviation in the average difference between the peak angles measured, there was a lot of variability at this point, which was mainly due to not controlling for the manner and speed at which the subjects leaned forward. Since this would not be controlled in real life situations either, adding a gyroscope or another accelerometer may be necessary if the accuracy of the trunk tilt estimates adversely affected performance of the control system. Simply low-pass filtering the existing control signal may introduce undesirable time delays when triggering stimulation to prevent falls.

The selection of a wireless wearable accelerometer as a tilt sensor for the trunk is just one of many possible choices for feedback sensors. It was selected because it was small, inexpensive, and already incorporated into many commercially available products (such as the Chronos sport watch used in this study). While only one axis was used for control in this study, the sensor is threedimensional and the control system could readily be extended to lateral bending in the coronal plane and recover from falls to the front, left, right, or intermediate directions.

\section{CONCLUSIONS}

This feasibility study shows that a simple thresholdbased trunk tilt controller can be used to modulate stimulation of the trunk and hip extensor muscles of individuals with SCI to restore seated posture during a forward fall. The controller worked consistently across all subjects in spite of considerable intrasubject variability in terms of injury level, voluntary and stimulated strength, and preserved sensory and motor function. This study demonstrated that a sensor-based threshold controller can work reliably under laboratory conditions, and continued testing is needed to demonstrate the clinical significance of these simple control systems at home and community environments. Initial performance of the threshold-based controller for restoring upright, seated posture provides a solid foundation upon which to base the development of more complex control schemes to improve seated balance, manual wheelchair propulsion, and dynamic trunk stability and mobility during activities of daily living for individuals living with SCI.

\section{ACKNOWLEDGMENTS}

\section{Author Contributions:}

Study concept and design: M. L. Audu, R. J. Triolo, J. O. Murphy. Acquisition of data: J. O. Murphy, L. M. Lombardo, K. M. Foglyano. Analysis and interpretation of data: J. O. Murphy, M. L. Audu, R. J. Triolo.

Drafting of manuscript: J. O. Murphy.

Statistical analysis: J. O. Murphy.

Obtained funding: M. L. Audu, R. J. Triolo. 
Administrative, technical, or material support: L. M. Lombardo, K. M. Foglyano.

Study supervision: M. L. Audu, R. J. Triolo.

Financial Disclosures: The authors have declared that no competing interests exist.

Funding Sources: This material was based on work supported in part by the Department of Veterans Affairs Rehabilitation Research and Development Service (grant B6406R) and the Department of Defense SCI Research Program (grant W81XWH-10-2-0141).

Additional Contributions: The authors would like to thank the volunteers who participated in this study, as well as John Schnellen-

berger, Jeremy Dunning, Michael Miller, Stephanie Nogan Bailey, and Nathan Makowski for their assistance with this study.

Institutional Review: All subjects signed the consent forms approved by the institutional review board of the Louis Stokes Cleveland Department of Veterans Affairs Medical Center.

Participant Follow-Up: The authors plan to inform participants of the publication of this study.

\section{REFERENCES}

1. Kukke SN, Triolo RJ. The effects of trunk stimulation on bimanual seated workspace. IEEE Trans Neural Syst Rehabil Eng. 2004;12(2):177-85. [PMID:15218932] http://dx.doi.org/10.1109/TNSRE.2004.827222

2. Kemmoku T, Furumachi K, Shimamura T. Force on the sacrococcygeal and ischial areas during posterior pelvic tilt in seated posture. Prosthet Orthot Int. 2013;37(4):282-88. [PMID:23169902] http://dx.doi.org/10.1177/0309364612465429

3. Sinnott KA, Milburn P, McNaughton H. Factors associated with thoracic spinal cord injury, lesion level and rotator cuff disorders. Spinal Cord. 2000;38(12):748-53.

[PMID:11175375]

http://dx.doi.org/10.1038/sj.sc.3101095

4. Akbar M, Balean G, Brunner M, Seyler TM, Bruckner T, Munzinger J, Grieser T, Gerner HJ, Loew M. Prevalence of rotator cuff tear in paraplegic patients compared with controls. J Bone Joint Surg Am. 2010;92(1):23-30.

[PMID:20048092]

http://dx.doi.org/10.2106/JBJS.H.01373

5. Anderson KD. Targeting recovery: Priorities of the spinal cord-injured population. J Neurotrauma. 2004;21(10): 1371-83. [PMID:15672628] http://dx.doi.org/10.1089/neu.2004.21.1371

6. Seelen HA, Potten YJ, Huson A, Spaans F, Reulen JP. Impaired balance control in paraplegic subjects. J Electromyogr Kinesiol. 1997;7(2):149-60. [PMID:20719700] http://dx.doi.org/10.1016/S1050-6411(97)88884-0

7. Hobson DA, Tooms RE. Seated lumbar/pelvic alignment. A comparison between spinal cord-injured and noninjured groups. Spine. 1992;17(3):293-98. [PMID:1566167] http://dx.doi.org/10.1097/00007632-199203000-00009
8. Zacharkow D. Posture: Sitting, standing, chair design, and exercise. Springfield (IL): Thomas; 1988.

9. Andersson GB, Ortengren R, Nachemson A. Intradiskal pressure, intra-abdominal pressure and myoelectric back muscle activity related to posture and loading. Clin Orthop Relat Res. 1977;(129):156-64. [PMID:608269] http://dx.doi.org/10.1097/00003086-197711000-00018

10. Andersson BJ, Ortengren R, Nachemson AL, Elfström G, Broman H. The sitting posture: An electromyographic and discometric study. Orthop Clin North Am. 1975;6(1):105-20. [PMID:1113963]

11. Ham R, Aldersea P, Porter D. Wheelchair users and postural seating: A clinical approach. London (United Kingdom): Churchill Livingstone; 1998.

12. Cooper RA. Wheelchair selection and configuration. New York (NY): Demos; 1998.

13. DeLisa JA, Gans BM, Walsh NE. Physical medicine and rehabilitation: Principles and practice. Philadelphia (PA): Lippincott Williams \& Wilkins; 2005.

14. Janssen-Potten YJ, Seelen HA, Drukker J, Reulen JP. Chair configuration and balance control in persons with spinal cord injury. Arch Phys Med. 2000;81(4):401-8. [PMID:10768527] http://dx.doi.org/10.1053/mr.2000.3859

15. Triolo RJ, Boggs L, Miller M, Nemuanitis G, Nagy J, Bailey SN. Implanted electrical stimulation of the trunk for seated postural stability and function after cervical spinal cord injury: A single case study. Arch Phys Med Rehabil. 2009;90(2):340-47. [PMID:19236990] http://dx.doi.org/10.1016/j.apmr.2008.07.029

16. Wu GA, Lombardo LM, Triolo RJ, Bogie KM. The effects of combined trunk and gluteal neuromuscular electrical stimulation on posture and tissue health in spinal cord injury. PM\&R. 2013;5(8):688-96. [PMID:23542776]

http://dx.doi.org/10.1016/j.pmrj.2013.03.025

17. Triolo RJ, Bailey SN, Lombardo LM, Miller ME, Foglyano $\mathrm{K}$, Audu ML. Effects of intramuscular trunk stimulation on manual wheelchair propulsion mechanics in 6 subjects with spinal cord injury. Arch Phys Med Rehabil. 2013;94(10): 1997-2005. [PMID:23628377]

http://dx.doi.org/10.1016/j.apmr.2013.04.010

18. Triolo RJ, Bailey SN, Miller ME, Lombardo LM, Audu ML. Effects of stimulating hip and trunk muscles on seated stability, posture, and reach after spinal cord injury. Arch Phys Med Rehabil. 2013;94(9):1766-75.

[PMID:23500182]

http://dx.doi.org/10.1016/j.apmr.2013.02.023

19. Vanoncini M, Holderbaum W, Andrews BJ. Electrical stimulation for trunk control in paraplegia: A feasibility study. Control Eng Pract. 2012;20(12):1247-58. http://dx.doi.org/10.1016/j.conengprac.2012.06.007 
20. Kirshblum SC, Burns SP, Biering-Sorensen F, Donovan W, Graves DE, Jha A, Johansen M, Jones L, Krassioukov A, Mulcahey MJ, Schmidt-Read M, Waring W. International standards for neurological classification of spinal cord injury (revised 2011). J Spinal Cord Med. 2011;34(6):535-46.

[PMID:22330108] http://dx.doi.org/10.1179/204577211X13207446293695

21. Smith B, Peckham PH, Keith MW, Roscoe DD. An externally powered, multichannel, implantable stimulator for versatile control of paralyzed muscle. IEEE Trans Biomed Eng. 1987;BME-34(7):499-508. [PMID:3610200] http://dx.doi.org/10.1109/TBME.1987.325979

22. Davis JA Jr, Triolo RJ, Uhlir J, Bieri C, Rohde L, Lissy D, Kukke S. Preliminary performance of a surgically implanted neuroprosthesis for standing and transfers - where do we stand? J Rehabil Res Dev. 2001;38(6):609-17. [PMID:11767968]

23. Triolo RJ, Bailey SN, Miller ME, Rohde LM, Anderson JS, Davis JA Jr, Abbas JJ, DiPonio LA, Forrest GP, Gater DR Jr, Yang LJ. Longitudinal performance of a surgically implanted neuroprosthesis for lower-extremity exercise, standing, and transfers after spinal cord injury. Arch Phys Med Rehabil. 2012;93(5):896-904. [PMID:22541312] http://dx.doi.org/10.1016/j.apmr.2012.01.001

24. Mushahwar VK, Jacobs PL, Normann RA, Triolo RJ, Kleitman N. New functional electrical stimulation approaches to standing and walking. J Neural Eng. 2007;4(3):S181-97. [PMID:17873417] http://dx.doi.org/10.1088/1741-2560/4/3/S05

25. Smith B, Tang Z, Johnson MW, Pourmehdi S, Gazdik MM, Buckett JR, Peckham PH. An externally powered, multichannel, implantable stimulator-telemeter for control of paralyzed muscle. IEEE Trans Biomed Eng. 1998;45(4): 463-75. [PMID:9556963] http://dx.doi.org/10.1109/10.664202

26. Nataraj R, Audu ML, Triolo RJ. Comparing joint kinematics and center of mass acceleration as feedback for control of standing balance by functional neuromuscular stimulation. J Neuroeng Rehabil. 2012;9:25. [PMID:22559852] http://dx.doi.org/10.1186/1743-0003-9-25

27. Nataraj R, Audu ML, Kirsch RF, Triolo RJ. Trunk acceleration for neuroprosthetic control of standing: A pilot study. $\mathrm{J}$ Appl Biomech. 2012;28(1):85-92. [PMID:21975251]

28. Nataraj R, Audu ML, Triolo RJ. Center of mass acceleration feedback control of standing balance by functional neuromuscular stimulation against external postural perturbations. IEEE Trans Biomed Eng. 2013;60(1):10-19. [PMID:22987499] http://dx.doi.org/10.1109/TBME.2012.2218601

29. Fisher LE, Miller ME, Bailey SN, Davis JA Jr, Anderson JS, Rhode L, Tyler DJ, Triolo RJ. Standing after spinal cord injury with four-contact nerve-cuff electrodes for quadriceps stimulation. IEEE Trans Neural Syst Rehabil Eng.
2008;16(5):473-78. [PMID:18990650]

http://dx.doi.org/10.1109/TNSRE.2008.2003390

30. Fisher LE, Tyler DJ, Anderson JS, Triolo RJ. Chronic stability and selectivity of four-contact spiral nerve-cuff electrodes in stimulating the human femoral nerve. J Neural Eng. 2009;6(4):046010. [PMID:19602729] http://dx.doi.org/10.1088/1741-2560/6/4/046010

31. Memberg WD, Peckham PH, Keith MW. A surgicallyimplanted intramuscular electrode for an implantable neuromuscular stimulation system. IEEE Trans Biomed Eng. 1994;2(2):80-91.

32. Davis JA Jr, Triolo RJ, Uhlir JP, Bhadra N, Lissy DA, Nandurkar S, Marsolais EB. Surgical technique for installing an eight-channel neuroprosthesis for standing. Clin Orthop Relat Res. 2001;(385):237-52. [PMID:11302320] http://dx.doi.org/10.1097/00003086-200104000-00035

33. Sharma M, Marsolais EB, Polando G, Triolo RJ, Davis JA Jr, Bhadra N, Uhlir JP. Implantation of a 16-channel functional electrical stimulation walking system. Clin Orthop Relat Res. 1998;(347):236-42. [PMID:9520896]

34. Trier SC, Buckett JR, Campean A, Miller ME, Montague FW, Vrabec TL, Weisgarber JA. A modular external control unit for functional electrical stimulation. Proceedings of the 6th Annual Conference of the International Functional Electrical Stimulation Society; 2001 Jun 16-20; Cleveland, $\mathrm{OH}$. Cleveland $(\mathrm{OH})$ : International Functional Electrical Stimulation Society; 2001. p. 108.

35. Luczak S, Oleksiuk W, Bodnicki M. Sensing tilt with MEMS accelerometers. IEEE Sens J. 2006;6(6):1669-75. http://dx.doi.org/10.1109/JSEN.2006.881433

36. Bliley KE, Kaufman KR, Gilbert BK. Methods for validating the performance of wearable motion-sensing devices under controlled conditions. Meas Sci Technol. 2009; 20(4):1-13.

http://dx.doi.org/10.1088/0957-0233/20/4/045802

37. Thrasher TA, Sin VW, Masani K, Vette AH, Craven BC, Popovic MR. Responses of the trunk to multidirectional perturbations during unsupported sitting in normal adults. J Appl Biomech. 2010;26(3):332-40. [PMID:20841625]

38. Fujita K, Hoshimiya N, Handa Y, Ichie M. Trunk stabilization by feedback sway control in FES-induced paraplegic standing. Proceedings of the 17th Annual Conference of the IEEE Engineering in Medicine and Biology Society; 1995 Sep 20-23; Montreal, Quebec. Piscataway (NJ): The Institute of Electrical and Electronics Engineers; 1997. p. 1141-42.

39. Djurić-Jovičić MD, Jovičić NS, Popović DB. Kinematics of gait: New method for angle estimation based on accelerometers. Sensors (Basel Switzerland). 2011;11(11):10571-85. [PMID:22346659] http://dx.doi.org/10.3390/s111110571

40. Boonstra MC, van der Slikke RM, Keijsers NL, van Lummel $\mathrm{RC}$, de Waal Malefijt MC, Verdonschot N. The accuracy of 
measuring the kinematics of rising from a chair with accelerometers and gyroscopes. J Biomech. 2006;39(2):354-58.

[PMID:16321638]

http://dx.doi.org/10.1016/j.jbiomech.2004.11.021

41. Mayagoitia RE, Nene AV, Veltink PH. Accelerometer and rate gyroscope measurement of kinematics: An inexpensive alternative to optical motion analysis systems. J Biomech. 2002;35(4):537-42. [PMID:11934425]

http://dx.doi.org/10.1016/S0021-9290(01)00231-7

Submitted for publication September 9, 2013. Accepted in revised form January 22, 2014.

This article and any supplementary material should be cited as follows:

Murphy JO, Audu ML, Lombardo LM, Foglyano KM,
Triolo RJ. Feasibility of closed-loop controller for righting seated posture after spinal cord injury. J Rehabil Res Dev. 2014;51(5):747-60.

http://dx.doi.org/10.1682/JRRD.2013.09.0200

ResearcherID/ORCID: Julie O. Murphy, BSE: H-32682014; Musa L. Audu, PhD: H-3251-2014; Lisa M. Lombardo, MPT: H-3691-2014; Kevin M. Foglyano, BSE: C9888-2013; Ronald J. Triolo, PhD: H-3712-2014 